\title{
Ensino da fotografia no âmbito da educação superior em Design: uma revisão sistemática de literatura
}

\author{
Photography education in Design higher education courses: a systematic literature \\ review
}

AMARAL, Ana Leticia Oliveira do; Mestranda; Universidade Federal de Santa Catarina - UFSC amaral.analeticia@gmail.com

GONÇALVES, Berenice Santos; Doutora; Universidade Federal de Santa Catarina - UFSC

berenice.santos@cce.ufsc.br

GITIRANA, Marcelo; Doutor; Universidade do Estado de Santa Catarina - UDESC

marcelo.gitirana@gmail.com

\section{Resumo}

A fotografia tem uma utilização fundamental e extensiva no estudo de diversas áreas do conhecimento, possui uma estreita relação com as atividades do Design e constitui-se primordial à produção cultural contemporânea. Nesse sentido, o ensino da fotografia requer uma inovação constante para manter a sua relevância, visto que dificilmente, técnicas convencionais de ensino, envolvam as gerações que já cresceram em uma era digital. Diante disso, este estudo se propôs a identificar e analisar pesquisas sobre o ensino da fotografia no contexto de ensino superior. Para tanto, adotou-se como método uma revisão sistemática de literatura, que permite sintetizar o conhecimento disponível sobre um determinado assunto e direcioná-lo. A partir dos resultados alcançados nesse processo, são apresentados os principais apontamentos encontrados. Ao final, fica evidente que o reduzido número de publicações envolvendo preocupações com os processos de ensino da fotografia indicam uma necessidade de ampliação de pesquisas com essa temática.

Palavras Chave: ensino da fotografia; ensino superior; blended learning e revisão sistemática.

\begin{abstract}
Photography has a fundamental and extensive use in the study of several areas of knowledge, has a close relationship with the activities of Design and is essential to contemporary cultural production. In this sense, the teaching of photography requires constant innovation to maintain its relevance, since conventional teaching techniques hardly involve the generations that have already grown up in a digital age. Therefore, this study aimed to identify and analyze research on the teaching of photography in the context of higher education. For that, a systematic literature review was adopted as a method, which allows synthesizing the available knowledge about a given subject and direct it. From the results achieved in this process, the main notes are presented. In the end, it is evident that the reduced number of publications involving preoccupations with the teaching processes of the photography indicate a need to expand research on this subject.
\end{abstract}

Keywords: photographic education; higher education; blended learning and systematic review. 


\section{Introdução}

Produzir imagens faz parte da evolução da humanidade. Pode-se dizer que hoje a imagem fotográfica faz parte do cotidiano do homem contemporâneo, seja informando, formando ou impulsionando à reflexão (SOBRAL, 2011). Na sociedade atual a imagem fotográfica possui uma presença ubíqua, onde diversos aparatos capturam e compartilham milhões de imagens a todo o momento em todo o mundo (HAND, 2012; SANTAELLA, 2014; MANOVICH, 2017). As funcionalidades dos equipamentos tecnológicos se unem para potencializar a mobilidade e a interatividade dos conteúdos, mudando as relações sociais, tecnológicas, econômicas e industriais. Entretanto, apesar da imagem fotográfica ter sido trivializada na atualidade (SANTAELLA, 2014), ela tem uma utilização fundamental e extensiva no estudo de diversas áreas do conhecimento (RUBINSTEIN, 2009), possui uma estreita relação com as atividades do Design e constitui-se primordial à produção cultural contemporânea.

Nesse sentido, Sobral (2011, p. 137) afirma que os "novos fluxos midiáticos estão impondo mudanças no exercício do ensino, da produção e do consumo de imagens, além de colocarem à prova as teorias elaboradas até agora sobre a imagem fotográfica e suas práticas". Mendonza (2015) reforça que o rápido avanço tecnológico faz primordial a necessidade de professores, alunos e profissionais manterem-se atualizados com as inovações tecnológicas. Demandando, além de uma constante atualização, o controle cuidadoso das informações adquiridas, já que "isso afeta tanto os currículos quanto às metodologias e estratégias aplicadas em sala de aula" (p. 148).

Abrahmov e Ronen (2008) afirmam que o objetivo do ensino de fotografia é desenvolver habilidades tanto de "leitura" quanto de "escrita" de imagens. Para os autores, explorar o significado de uma fotografia deve ser mais parecido com a leitura de uma frase complexa do que com a leitura de uma única palavra. E segundo Rivera (2012) aprender a tirar fotos é selecionar um olhar entre muitos olhares possíveis, dessa maneira, adquirindo os critérios necessários para selecionar um ponto de vista no espaço e no tempo. Ao se tornarem criadores e leitores de imagens, os estudantes aprendem habilidades de comunicação e resolução de problemas tornando-se, assim, mais aptos a navegar na cultura cada vez mais visual.

Portanto, o ensino da fotografia requer uma inovação constante para manter a relevância frente a cultura contemporânea, visto que dificilmente, métodos convencionais de ensino, envolvam as gerações que já cresceram em uma era digital, onde a autoaprendizagem e a exploração ativa da informação são tarefas diárias (NEWBURRY, 1997; ROBBINS, 2000; FAKRI; YUSOFF; SAID, 2015; ZHANG et al, 2013). Sendo assim, a incorporação de tecnologias digitais no ensino é fundamental para democratizar o acesso a uma educação superior de qualidade e equidade, ampliar a interiorização e melhorar os processos de ensino e aprendizagem (DIRETRIZES DE POLÍTICA PÚBLICA PARA O ENSINO SUPERIOR BRASILEIRO, 2017).

Nessa perspectiva, cabe retomar a afirmativa de Tori (2010) que diz que a cooperação entre métodos e técnicas presenciais e virtuais no ensino podem auxiliar a garantir um melhor acompanhamento do desenvolvimento da aprendizagem, incentivar práticas colaborativas, motivar e envolver os alunos. Dessa forma, segundo o relatório NMC Horizon Report (2017), a tendência Blended Learning, atualmente, se tornou um termo abrangente que engloba qualquer combinação de ensino tradicional com modos de instrução facilitados pela tecnologia, que capacitem o corpo docente com uma variedade de ferramentas para atender às diferentes necessidades dos estudantes. 
Assim considerando o contexto exposto, este estudo se propôs a identificar e analisar pesquisas sobre o ensino da fotografia no contexto de ensino superior, assim como, destacar, dentre as pesquisas encontradas materiais e tecnologias que estão sendo utilizados ou desenvolvidos para o ensino-aprendizagem dos conceitos de fotografia. Para atingir este objetivo, realizou-se uma Revisão Sistemática de Literatura. Este método adota um processo replicável, científico e transparente e se dá por meio de buscas exaustivas em estudos publicados em base de dados, assim como, fornece um registro das decisões, procedimentos e conclusões dos pesquisadores (WEBSTER; WATSON, 2002; TRANFIELD; DENYER; SMART, 2003; LEVY; ELLIS, 2006).

\section{Procedimentos Metodológicos}

Este estudo realizou uma Revisão Sistemática de Literatura (RSL) de caráter amplo e exploratório. Conforme Sampaio e Mancini (2007) este tipo de investigação científica fornece evidências relacionadas a uma estratégia de intervenção específica por meio de uma aplicação de método de pesquisa explícito e sistematizado, apreciação crítica e síntese da informação selecionada. Além disso, é importante que seja executada de forma confiável, realizada de maneira sistemática e de modo compreensivo (WEBSTER; WATSON, 2002; LEVY; ELLIS, 2006). Para esta pesquisa utilizou-se o método proposto por Sampaio e Mancini (2007), que descrevem o desenvolvimento da RSL em cinco etapas, a saber, (I) definir a pergunta, (II) buscar a evidência, (III) revisar e selecionar os estudos, (IV) analisar a qualidade metodológica dos estudos e (V) apresentar os resultados, como pode ser visto na figura 1.

Figura 1 - Etapas da revisão sistemática

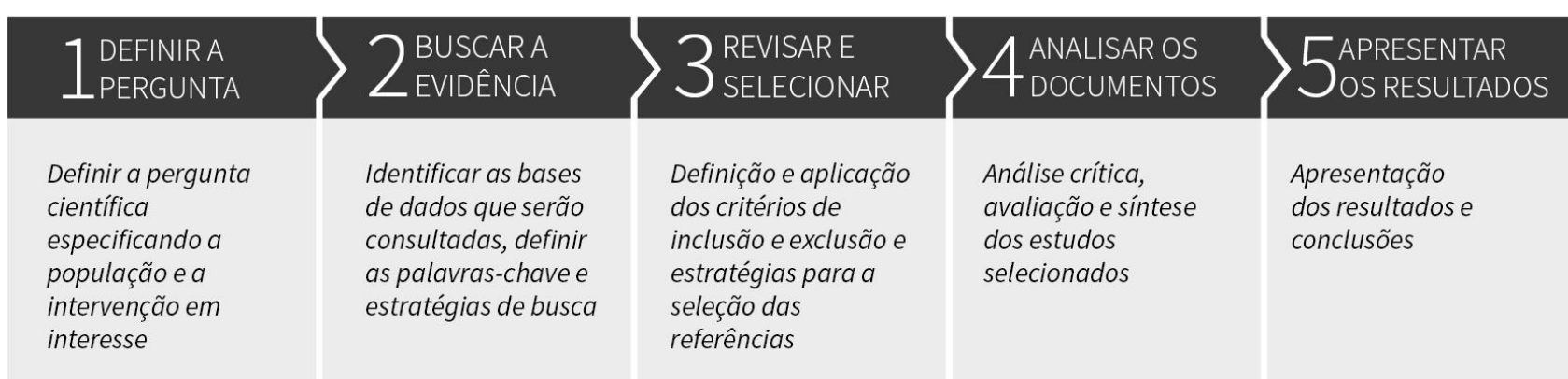

Fonte: elaborado pelos autores com base em Sampaio e Mancini (2007)

Definida a questão de pesquisa - identificar pesquisas atuais sobre o ensino da fotografia no âmbito do ensino superior - com base no método proposto a estratégia de busca foi desenvolvida a partir da definição de dois eixos relevantes ao estudo: ensino da fotografia e ensino superior. Entretanto, antes de definir os dois eixos finais, foram testados quatro (4) diferentes eixos para as buscas das referências que incluíam as palavras "ensino-fotografia-designtecnologias digitais", contudo devido ao reduzido número de resultados optou-se por utilizar apenas os dois eixos anteriormente definidos. 0 quadro 1, a seguir, mostra as alternativas geradas de termos relacionados, todos em inglês, para a definição final da string ${ }^{1}$ de busca.

\footnotetext{
${ }^{1}$ Conjunto de palavras e termos referentes ao tema da pesquisa conectados por operadores lógicos AND e OR (FABBRI; OCTAVIANO; HERNANDES, 2017).
} 
Quadro 1 - Relação das palavras-chave

\begin{tabular}{ll}
\hline Ensino da Fotografia & Ensino Superior \\
\hline Photography & Higher education \\
Photography education & Undegraduate \\
Teaching photography & Instruction \\
Photographic techniques &
\end{tabular}

Fonte: elaborado pelos autores (2018)

Identificadas as palavras-chave e termos referentes ao tema de pesquisa, para desenvolver a string de busca é preciso compreender a utilização de operadores lógicos comumente aplicados em buscas avançadas ou em buscas booleanas (CONFORTO; AMARAL; SILVA, 2011). No desenvolvimento da string houve certa dificuldade na escolha dos termos relacionados que melhor se adequavam, visto que a expressão "ensino da fotografia" por vezes se confundia com fotografia para o ensino. Contudo, como "a construção da string segue um processo de definição, teste e adaptação" (CONFORTO; AMARAL; SILVA, 2011), foram testadas diversas combinações de termos relacionados às palavras-chave até se chegar ao arranjo que gerou um resultado mais satisfatório ao escopo desta pesquisa.

Dessa maneira, para ligar os termos sinônimos de cada eixo, foi utilizado o operador booleano "OR", já para ligar os dois eixos "ensino da fotografia" e "ensino superior" o operador "AND" foi empregado. Além disso, optou-se por utilizar o asterisco no radical das palavras "photograph*" e "technique*" para alcançar mais variações nominais. Assim, a string final de busca foi definida como: (photograph* OR "photograph* education" OR "teaching photograph*" $O R$ "photograph* technique*") AND ("higher education" OR undergraduate OR instruction).

A partir disso, a busca das evidências se deu em três bases de dados internacionais, sendo elas Scopus, Web of Science e ProQuest. Além da recorrência na área de Ciências Sociais Aplicadas as bases foram selecionadas devido aos seguintes critérios:

- Scopus: é o maior banco de dados de resumos e citações de literatura revisada por pares: revistas científicas, livros e trabalhos de conferência. Contém mais de 15 mil periódicos indexados (SCOPUS, 2017).

- Web of Science: uma base de dados multidisciplinar com acesso a referências e resumos em todas as áreas do conhecimento. Cobre aproximadamente 12 mil periódicos (WEB OF SCIENCE, 2017).

- ProQuest: é uma base de dados que contém artigos de periódicos, dissertações, teses, documentos técnicos entre outros (PROQUEST, 2017).

A busca ocorreu em dois dias consecutivos (12 e 13 de dezembro de 2017), com os eixos dispostos na string apresentada abaixo, identificando-se os seguintes resultados: 
Quadro 2 - Resultados numéricos da busca

\begin{tabular}{lcccc}
\hline Ensino da Fotografia & Scopus & Proquest & WoS & Total \\
\hline $\begin{array}{l}\text { (photograph* OR "photograph* education" OR } \\
\text { "teaching photograph*" OR "photograph* technique*") }\end{array}$ & 799 & 693 & 744 & 2236 \\
AND ("higher education" OR undergraduate OR & & & & \\
instruction) & & & &
\end{tabular}

Fonte: elaborado pelos autores (2018)

Foram buscados artigos de periódicos publicados ou aceitos para publicação, bem como artigos de anais de congressos sem restrição de ano de publicação, nem especificação de área. Ressalta-se que todas as bases foram acessadas por meio do sistema CAPES. Quanto ao idioma, foram selecionadas apenas referências escritas em português, espanhol e inglês. Em relação ao acesso, foram mantidos apenas os artigos acessíveis gratuitamente por meio do: (1) Portal de Periódicos da CAPES na instituição Universidade Federal de Santa Catarina - UFSC; (2) Google Acadêmico; (3) Portal das editoras e (4) através do sistema COMUT na instituição envolvida.

Para o processo de seleção, as referências encontradas nas bases de dados foram exportadas - com todas as suas informações - nos formatos .BibTEX e .RIS para, em seguida, serem importadas no gerenciador de referências Mendeley, que organiza os documentos em uma tabela com diversas informações como autor, título do artigo, ano e local de publicação. Além disso, esta ferramenta permite a verificação da existência e exclusão de referências duplicadas entre as buscas.

Pesquisas que abordavam no seu escopo questões sobre a inserção da fotografia no ensino proporcionado por escolas, e pesquisas que tratam a fotografia como ferramenta auxiliar nos processos de ensino, foram excluídas por não se enquadrarem ao escopo desse estudo. Assim, foi aplicada a primeira filtragem constituída pela leitura do título, resumo e palavras-chave dos artigos encontrados. Após a exclusão dos documentos que não tinham relação com o tema, foi realizada a leitura dos artigos na íntegra. O critério de seleção dos trabalhos foi o nível de contribuição para responder à pergunta de pesquisa proposta. Dessa forma, obteve-se um portfólio com 20 artigos, contudo 9 referências foram excluídas, visto que não foi possível encontrar o arquivo de duas publicações e as demais não se adequavam aos critérios estabelecidos nesta pesquisa. A figura 2 sintetiza o processo de filtragem das referências. 
Figura 2 - Processo de seleção dos documentos

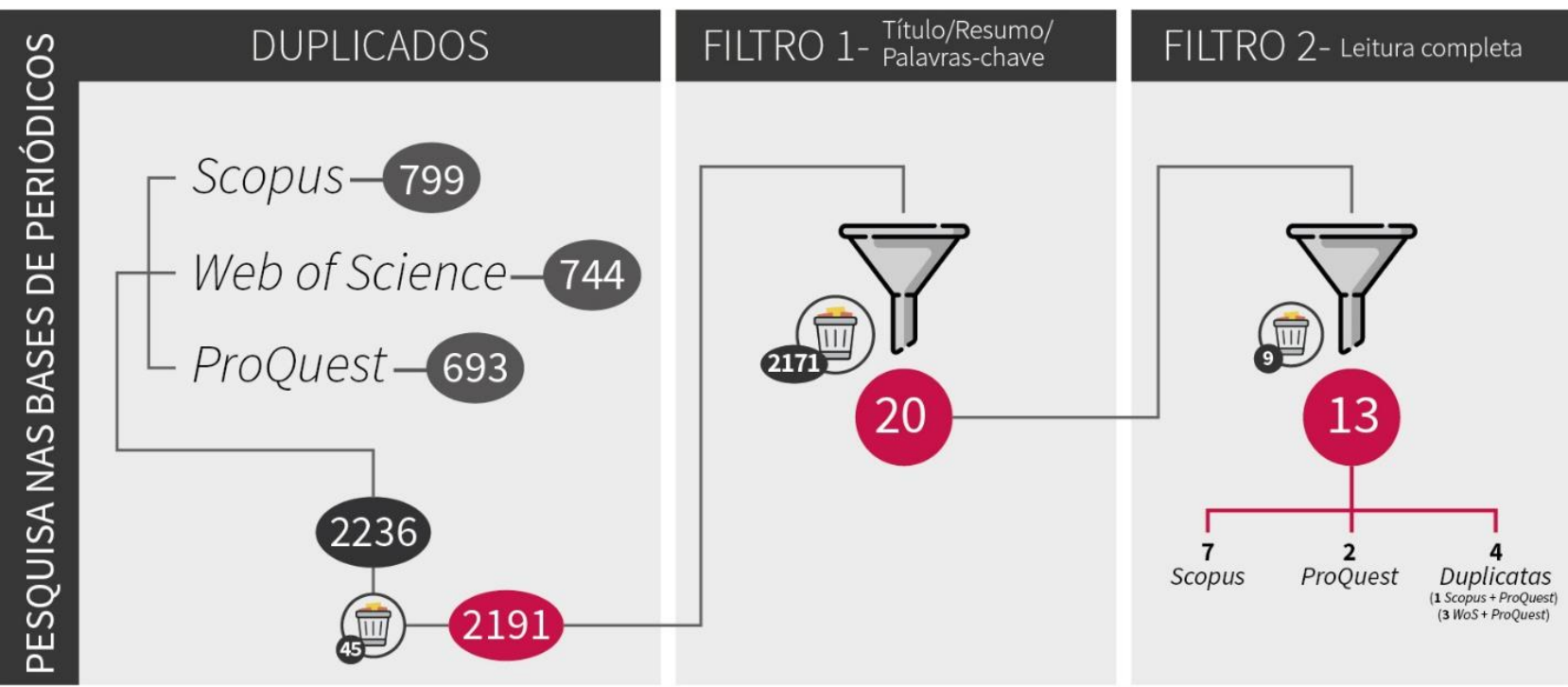

Fonte: elaborado pelos autores (2018)

\section{Resultados}

Assim, o portfólio final de referências apresenta um total de treze (13) publicações, sendo sete (7) oriundas da base de dados Scopus, duas (2) da ProQuest e quatro (4) artigos que aparecem em mais de uma base. $\mathrm{O}$ quadro 3 apresenta a referência completa dos estudos e a base de dados em que foram encontrados.

Quadro 3 - Portfólio final dos 13 trabalhos selecionados. Legenda: (A) Article, (CP) Conference Paper

\begin{tabular}{|c|c|c|c|c|}
\hline \multirow[b]{2}{*}{ Referência } & \multirow[b]{2}{*}{ Tipo } & \multicolumn{3}{|c|}{ Base } \\
\hline & & $\begin{array}{l}n \\
\frac{n}{0} \\
\text { 心 }\end{array}$ & $\begin{array}{l}\text { पू } \\
\frac{0}{2} \\
\frac{0}{0}\end{array}$ & ณ̊ \\
\hline
\end{tabular}

MANEEWAN, N, V. S.; LERTYOSBORDIN, C. A development of knowledge management process on cloud computing to support creative problem-solving skill on studio photography for undergraduate students. 6th International Conference on Industrial Technology and Management, ICITM 2017, p. 27-31, 2017.

PHUNGSUK, R.; VIRIYAVEJAKUL, C.; RATANAOLARN, T. Development of a problembased learning model via a virtual learning environment. Kasetsart Journal of Social Sciences, v. 38, n. 3, p. 297-306, 2017.

2016

SUKSAI, P. The Effectiveness and the Comparison of e-Learning Lessons Efficiency on the Principles of Digital Photography in Technology of Photography Course. International Journal of Information and Education Technology, v. 6, n. 8, p. 598602, 2016. 
MORGADO-AGUIRRE, B.; LOPEZ-MARTIN, E.; CONESA-TEJADA, S. El uso de las TIC en la enseñanza universitaria de la fotografía. Primeros resultados del proyecto de innovación docente de la Universidad de Murcia. Arte, Individuo y Sociedad, v. 27, n. 2, p. 327-351, 2015.

FAKRI, S. H. M.; YUSOFF, A,; SAID, C. S. APPLYING GESTALT VARIABLES IN LEARNING PHOTOGRAPHY WITH VIRTUAL GAMES. Jurnal Teknologi, Johor Bahru, Malásia, v. 75, n. 3, p.111-115, 2015.

2013

ZHANG, Y.; HAO, X.; WANG, J.; JIAO, W.; DAI, W. The Thinking of Integrating between Informational Technology and Photography Course. International Journal of Digital Content Technology and its Applications, v. 7, n. 2, p. 487-494, 2013.

2012

RIVERA, C. D. Enseñanza de la fotografía en ambientes virtuales de aprendizaje. Undécima Conferencia Iberoamericana en Sistemas, Cibernética e Informática, 2012.

CHIN, S. H.; LIEN, J. H.; HUANG, Y. X. Examining learning attitude toward ICT in digital photography. Lecture Notes in Electrical Engineering, v. 2, p. 491-497, 2012.

\section{1}

MARZAL, J.; SOLER, M. Consumption Patterns and Uses of Photography in Digital Era among Communication Students. Comunicar, v. 19, n. 37, p. 109-116, 2011.

\section{8}

ABRAHMOV, S. L.; RONEN, M. Double blending: online theory with on-campus practice in photography instruction. Innovations in Education and Teaching International, v. 45, n. 1, p. 3-14, 2008.

ROBBIE, D.; ZEENG, L. IT's evolving, they're changing, we're listening: everybody's learning. ASCILITE 2008 - The Australasian Society for Computers in Learning in Tertiary Education, p. 809-818, 2008.

\section{7}

VOISARD, R.; CHAMPOD, C.; FURRER, J.; CURCHOD, J.; VAUTIER, A.; MASSONNET, G.; BUZZINI, P. Nicephor[e]: A web-based solution for teaching forensic and scientific photography. Forensic Science International, v. 167, n. 3, p. 196-200, 2007.

2003

BROWN, S.; CRUICKSHANK, I. The virtual studio. International Journal of Art and Design Education, v. 22, n. 3, p. 281-288, 2003.
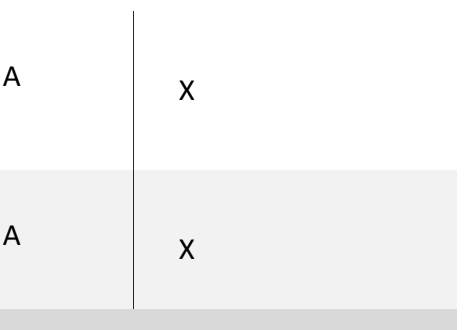

A

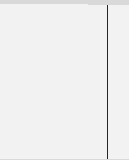

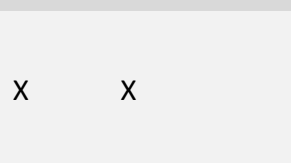

$\mathrm{CP}$

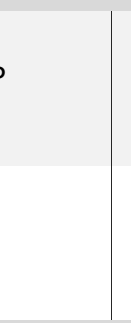

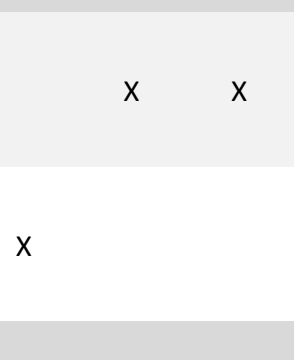

A

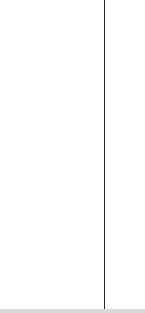
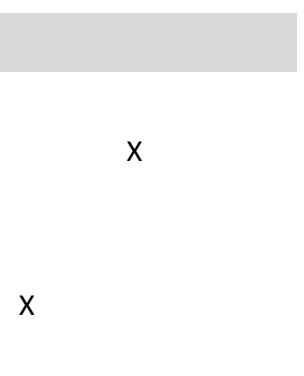

$x$

A

Fonte: elaborado pelos autores (2018)

\section{Discussões}

Os estudos concentram-se em melhorias no ensino da fotografia utilizando abordagens de aprendizagem combinado (blended learning designs ou b-learning), baseados no desenvolvimento de processos de gerenciamento do conhecimento e na resolução de problemas de práticas específicas de ensino (MANEEWAN; LERTYOSBORDIN, 2017; PHUNGSUK; VIRIYAVEJAKUL; RATANAOLARN, 2017), na utilização de redes sociais para potencialização do aprendizado 
(ROBBIE; ZEENG, 2008), no desenvolvimento de materiais específicos para estudo online (FAKRI; YUSOFF; SAID, 2015; ZHANG et al, 2013; BROWN; CRUICKSHANK, 2003), no desenvolvimento de ambientes virtuais de aprendizagem (ABRAHMOV; RONEN, 2008; VOISARD et al, 2007; RIVERA, 2012), assim como, na avaliação da utilização de ambientes de aprendizagem junto aos estudantes (SUKSAI, 2016; MORGADO-AGUIRRE; LOPEZ-MARTIN; CONESA-TEJADA, 2015; ZHANG et al, 2013; CHIN; LIEN; HUANG, 2012), além de um estudo comportamental sobre a percepção dos estudantes espanhóis de comunicação sobre a fotografia digital (MARZAL; SOLER, 2011).

É possível afirmar que técnicas convencionais de ensino, que englobam quadro, explanação, slides e uma pequena parte prática de fotografia (ZHANG et al, 2013), dificilmente envolvem as gerações que já cresceram em uma era digital, onde a autoaprendizagem e a exploração ativa de informações são tarefas comum (FAKRI; YUSOFF; SAID, 2015). De acordo com Robbie e Zeeng (2008), para satisfazer as expectativas de ensino e aprendizagem no ensino superior no século XXI, as mudanças curriculares devem enfatizar as boas características do ensino, a saber, conhecimento, organização, comunicação, entusiasmo, flexibilidade, empatia e respeito, assim como as qualidades da prática de ensino, ou seja, a reflexão, criatividade e tomada de riscos. Nesse sentido, Suksai (2016) ainda afirma que para melhorar o desempenho dos estudantes que são ensinados por e-learning, os professores precisam trocar de postura, no lugar de provedores de conhecimento é necessário assumir o papel de facilitadores, motivando os alunos a aprender e auxiliando-os nas atividades. Dessa maneira, dentre as pesquisas selecionadas no portfólio final da revisão sistemática, as que mais se destacaram por buscar estratégias que visam a integração do ensino de fotografia na educação superior com as tecnologias digitais são detalhadas nos próximos parágrafos.

O estudo relatado por Brown e Cruickshank (2003) apresenta o desenvolvimento de um estúdio virtual que visava suprir a falta de tempo dos professores e o aumento na quantidade de alunos matriculados na disciplina de fotografia na Universidade de Montfort (Leicester, UK). Para tal foi registrada uma série de fotografias que foram organizadas de forma que o aluno poderia controlar iluminação, posição dos elementos e configurações da câmera de forma virtual sem precisar utilizar um estúdio fotográfico físico. Porém, a troca das aulas presenciais por aulas a distância demonstrou uma série de problemas inesperados como o problema na organização e comprometimento dos alunos pela falta da presença de um tutor. Assim, cabe ressaltar que incorporar as tecnologias digitais da comunicação e informação na educação não resolve os problemas nem transforma a educação. É preciso desenvolver modelos de educação mais inclusivos, assim como, traçar estratégias de utilização das mídias (SOBRAL, 2011; HORIZON, 2017).

O projeto Nicephor[e], tratado na pesquisa de Voisard et al (2007), propõe uma série organizada de cursos modulares online sobre fotografia científica, forense e microscopia que pode ser estruturado de acordo com a necessidade de cada instituição. A arquitetura dessa estrutura de e-learning é baseada em três sistemas principais: um sistema de gerenciamento de conteúdo, um sistema de gerenciamento de aprendizado (Blackboard ou Moodle ${ }^{2}$ ) e um sistema de gerenciamento de arquivos digitais. O sistema resultante possibilita a criação de conteúdo no

\footnotetext{
${ }^{2}$ Moodle: é um sistema livre de gerenciamento para criação de cursos online. Esses sistemas são também chamados de Ambientes Virtuais de Aprendizagem (AVA) ou de Learning Management System (LMS).

Blackboard: é um sistema de gestão de aprendizagem proprietária e seu uso é geralmente limitado a educadores de instituições que pagam uma taxa a cada ano para assumir um contrato de licença para o seu uso
} 
idioma desejado, compartilhamento entre instituições e a escolha de abordagens de aprendizagem (blended learning ou completamente a distância). Contudo, o artigo não menciona estratégias de inserção dessa tecnologia no currículo das disciplinas.

Já a pesquisa desenvolvida por Diane Robbie e Lynette Zeeng em 2008, buscou empregar uma nova abordagem para o ensino da fotografia levando em consideração os aspectos da tecnologia digital, a propensão dos alunos à interação social e o envolvimento com as tecnologias Web 2.0. Para as autoras, a adoção de alguns aspectos das redes sociais nos métodos de ensinoaprendizagem pode trazer resultados positivos. Este estudo foi o único encontrado que integrou uma rede social ao ensino da fotografia dentro de uma graduação em Design. O ponto fundamental do artigo é a explicação sobre a dinâmica das aulas com introdução da tecnologia digital no currículo da disciplina de fotografia. A abordagem combinada envolveu uma palestra semanal de uma hora, uma aula de estúdio de duas horas, também foram realizadas tarefas semanalmente baseadas em práticas que incluíram reflexão individual, além de análise e crítica online de trabalhos enviados, para o perfil da turma na rede social Flickr, por todos os alunos matriculados na disciplina. As aulas e o estúdio incluíram aplicações teóricas e demonstrações práticas, revisão de contribuições online e modelagem do processo de crítica.

O modelo de instrução apresentado por Abrahmov e Ronen (2008) também visa integrar uma camada teórica de alfabetização visual em uma disciplina tradicional de fotografia prática, de uma graduação de Design, utilizando atividades online individuais e colaborativas. A incorporação de elementos online em cursos acadêmicos oferece várias maneiras de implementar métodos instrucionais tradicionais, bem como a capacidade de incorporar novas estratégias tornadas viáveis por este meio. Abrahmov e Ronen (2008) argumentam que o objetivo da pesquisa ao usar a web não foi substituir as atividades tradicionais do curso, mas introduzir novos objetivos de ensino que não poderiam ser alcançados em sala devido às limitações de tempo de aula e da definição do curso como sendo prático por natureza. Para os autores, o componente baseado na web desenvolvido permitiu envolver os alunos na atividade individual, onde cada aluno, em seu próprio tempo, pode desenvolver suas habilidades de leitura de imagens através de um conjunto orientado e focado de atividades, aprendendo com exemplos de pares.

Rivera (2012) busca responder ao questionamento de como pode ser possível conscientizar e desenvolver competência na composição de imagens fotográficas, utilizando ambientes digitais e de simulação. Dessa maneira, apresenta em seu estudo o curso virtual de Fotografia e Iluminação oferecido pelo Programa Especial de Admissão e Mobilidade Acadêmica (PEAMA) da Universidade Nacional da Colômbia. O curso consiste em quatro capítulos independentes com um exercício diferente para cada capítulo e utiliza a plataforma Blackboard para a administração de documentos, fóruns, avaliações e exercícios. O conteúdo do curso é desenvolvido em um documento multimídia em formato narrativo sequencial onde é sempre dada maior evidência as imagens. Para o autor, o impacto das tecnologias de informação e comunicação na educação torna-se mais significativo a medida que se incorpora as características e linguagens apropriadas ao meio.

Por fim, Fakri, Yousoff e Said (2015) propõem aprimorar as habilidades e o desempenho dos alunos de fotografia com o auxílio de seis variáveis da teoria da Gestalt aplicada em um jogo sério. Para os autores, um novo ambiente no processo de aprendizagem pode aumentar 0 interesse do aluno em aprender e auxilia no processo de compreensão. A comunicação entre aluno e educador na aula e no jogo virtual é diferente, porque, de acordo com os autores, o 
educador considera mais fácil abordar o estudante e identificar os problemas enfrentados durante a execução do jogo virtual. A partir desse levantamento, foi possível identificar as principais estratégias propostas pelos autores para a potencialização do ensino da fotografia no contexto de ensino superior. Esses resultados foram sistematizados conforme a figura 3, a seguir.

Figura 3 - Principais estratégias encontradas nas publicações

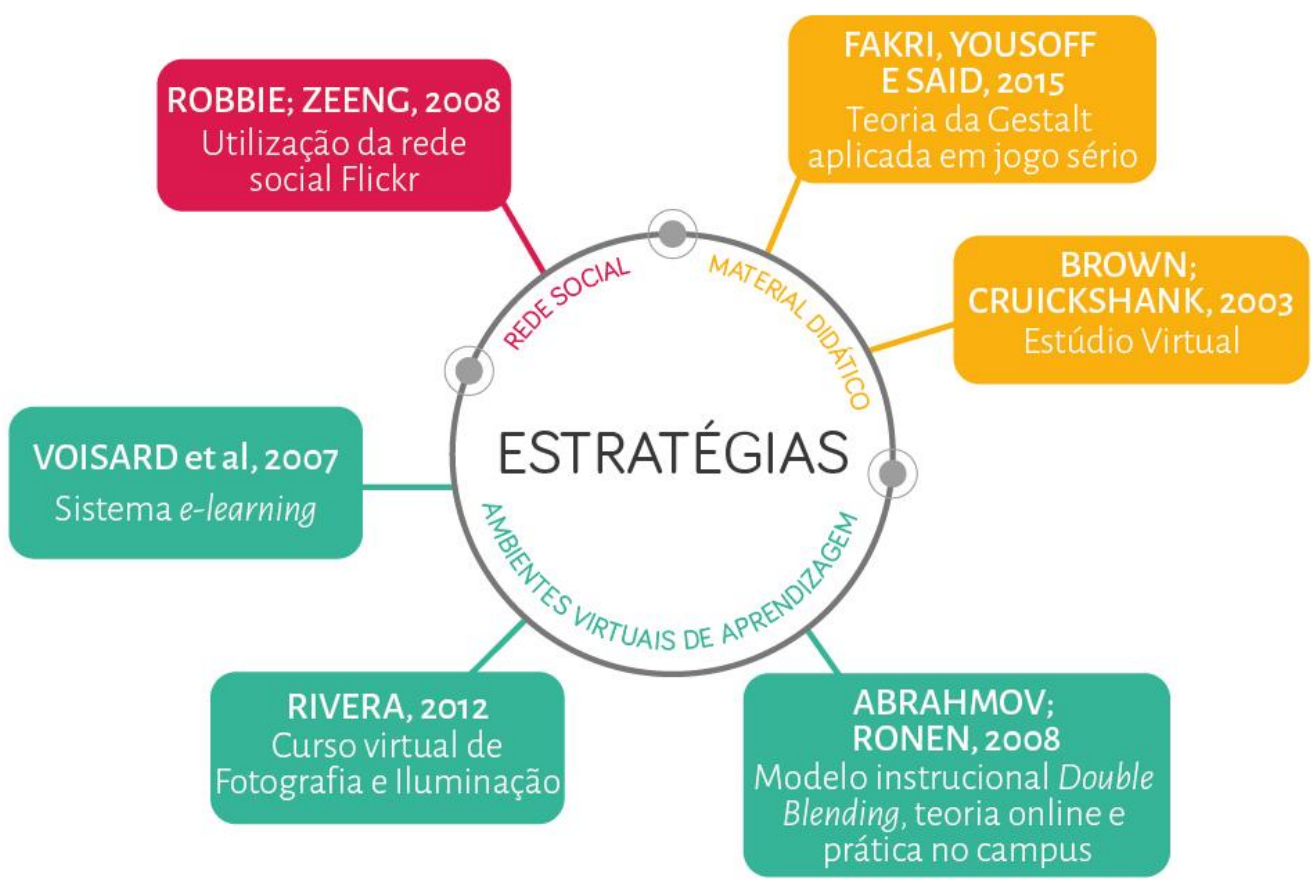

Fonte: elaborado pelos autores (2018)

Dessa maneira, é possível perceber que as estratégias desenvolvidas nas pesquisas encontradas estão concentradas em três eixos principais sendo o desenvolvimento de materiais didáticos e de ambientes virtuais de aprendizagem e na utilização de redes sociais. Com exceção dos estudos de Fakri et al (2015), que propôs a inserção de um jogo sério para aprendizagem da teoria da Gestalt dentro dos conteúdos abordados no ensino da fotografia. De Robbie e Zeeng (2008), que como abordado antes utilizaram o Flickr e o estudo de Brown e Cruickshank (2003) que desenvolveu um estúdio virtual para suprir o curto tempo proporcionado para a prática dos conhecimentos no estúdio fotográfico. As demais publicações trabalham com ambientes virtuais de aprendizagem construídos em plataformas como o Moodle e Blackboard (PHUNGSUK; VIRIYAVEJAKUL; RATANAOLARN, 2017; SUKSAI, 2016) ou desenvolvidos pelas equipes das universidades responsáveis (MANEEWAN; LERTYOSBORDIN, 2017; MORGADO-AGUIRRE; LOPEZMARTIN; CONESA-TEJADA, 2015; ZHANG et al, 2013; CHIN; LIEN; HUANG, 2012; ABRAHMOV; RONEN, 2008; VOISARD, 2007).

Ademais, com base nos resultados da RSL, é possível visualizar que os artigos são de origem geográfica dispersa, vale destacar que três (3) são originários da Tailândia e dois (2) da Espanha. Salienta-se ainda que nos países China, Colômbia, Taiwan, Israel, Suíça e Reino Unido foram encontradas apenas uma publicação cada. No que tange a evolução das publicações ao longo dos anos, percebe-se uma constante, entre anos sem e com produção, sendo os anos de 
2017, 2015, 2012 e 2008 com o maior número de publicações de duas para cada ano. Além disso, as pesquisas são oriundas de diferentes áreas do conhecimento, visto que cinco (5) dos artigos estão vinculados às ciências sociais, dois (2) às artes e humanidades, três (3) à engenharia, dois às ciências da computação e um (1) à área da saúde.

Diante disso, os resultados obtidos são de diversas áreas e buscam um objetivo comum, potencializar o ensino da fotografia frente às tecnologias digitais. Contudo, não foram encontradas pesquisas que buscam utilizar tecnologias como aplicativos mobile, realidade virtual ou realidade aumentada no ensino da fotografia. Dessa maneira, fica evidente que as pesquisas na área do ensino da fotografia necessitam buscar respaldo nos relatórios como o NMC Horizon que aponta novas tecnologias que podem auxiliar no processo de ensino-aprendizagem.

\section{Considerações finais}

Este estudo foi desenvolvido com o objetivo de identificar em pesquisas recentes como o ensino da fotografia, em instituições de educação superior, vem sendo abordado frente às tecnologias digitais da informação e comunicação. O levantamento realizado por meio da revisão sistemática de literatura mostrou-se objetivo e claro quanto aos procedimentos e resultados. Possibilitando assim a replicação do estudo para uma futura atualização. Quanto aos resultados, os artigos coletados apresentaram linhas de investigação correlatas e fundamentais para organizar um corpo de conhecimentos que possa contribuir com o ensino da fotografia, de acordo com os critérios deste estudo.

Uma das limitações encontradas na execução desta revisão sistemática diz respeito a definição das strings de busca, visto que diversas publicações encontradas utilizam como palavraschave ou indexadores os termos "fotografia", "ensino" e "educação" empregados separadamente. Dessa forma, para abarcar essas publicações foi necessário utilizar a palavra "photography" de modo isolada, gerando assim um expressivo número de resultados.

Portanto, apesar de inicialmente as buscas terem gerado um número expressivo de retornos, percebeu-se que muitos dos resultados não tinham relação direta com o tema ou não se adequavam ao escopo desta pesquisa. Além disso, considerando a utilização extensiva da imagem fotográfica em diversas áreas do conhecimento e da estreita relação com as atividades do Design, o reduzido número de publicações envolvendo preocupações com os processos de ensino da fotografia indicam uma necessidade de ampliação de pesquisas com essa temática.

Por fim, em concordância com as limitações encontradas no desenvolvimento dessa revisão sistemática, Marzal e Soler (2011) questionam as poucas investigações acerca da fotografia em revistas científicas, considerando a existência de um volume de produção e atividade fotográfica expressivo na vida das pessoas nos últimos anos.

\section{Referências}

ABRAHMOV, S. L.; RONEN, M. Double blending: online theory with on-campus practice in photography instruction. Innovations in Education and Teaching International, v. 45, n. 1, p. 3-14, 2008.

ADAMS BECKER, S.; CUMMINS, M., DAVIS, A.; FREEMAN, A.; HALL GIESINGER, C.; ANANTHANARAYANAN, V. (2017). NMC Horizon Report: 2017 Higher Education Edition. Austin, Texas: The New Media Consortium, 2017. 
BROWN, S.; CRUICKSHANK, I. The virtual studio. International Journal of Art and Design Education, v. 22, n. 3, p. 281-288, 2003.

CHIN, S. H.; LIEN, J. H.; HUANG, Y. X. Examining learning attitude toward ICT in digital photography. Lecture Notes in Electrical Engineering, v. 2, p. 491-497, 2012.

CONFORTO, Edivandro C.; AMARAL, Daniel C.; SILVA, Sérgio L. Roteiro para revisão bibliográfica sistemática: aplicação no desenvolvimento de produtos e gerenciamento de projetos. In: 8o Congresso Brasileiro de Gestão de Desenvolvimento de Produto, Porto Alegre, 12 a 14 set. 2011. Anais... p.1-12. Disponível em: <http://vision.ime.usp.br/ acmt/conforto.pdf>. Acesso em: 20 jan. 2018.

FABBRI, Sandra C. P. F.; OCTAVIANO, Fábio R.; HENANDES, Elis C. M. Protocolo da revisão sistemática. In: FELIZARDO, Katia R. et al. Revisão sistemática da literatura em engenharia de software: teoria e prática. Rio de Janeiro: Elsevier, 2017. p. 16-30.

FAKRI, S. H. M.; YUSOFF, A,; SAID, C. S. APPLYING GESTALT VARIABLES IN LEARNING PHOTOGRAPHY WITH VIRTUAL GAMES. Jurnal Teknologi, Johor Bahru, Malásia, v. 75, n. 3, p.111115, 2015.

HAND, Martin. Ubiquitous photography. Cambridge: Polity Press, 2012. 200 p.

LEVY, Yair; ELLIS, Timothy J. A system approach to conduct an effective literature review in support of information systems research. Informing Science Journal, v.9, p.181-212, 2006. Disponível em: < http://inform.nu/Articles/Vol9/V9p181-212Levy99.pdf>. Acesso em: 22 jan. 2018.

MANEEWAN, N, V. S.; LERTYOSBORDIN, C. A development of knowledge management process on cloud computing to support creative problem solving skill on studio photography for undergraduate students. In: 6th International Conference on Industrial Technology and Management, ICITM 2017, Anais... p. 27-31, 2017.

MANOVICH, Lev. Instagram and Contemporary Image. [S.L.]: [s.n.], 2017. 148 p. Disponível em: <http://manovich.net/content/04-projects/144-instagram-and-contemporaryimage/instagram_book_manovich.pdf>. Acesso em: 29 jan. 2018.

MARZAL, J.; SOLER, M. Consumption Patterns and Uses of Photography in Digital Era among Communication Students. Comunicar, v. 19, n. 37, p. 109-116, 2011.

MENDOZA, María Soledad. Sacar a la luz. Superando obstáculos en la enseñanza de la fotografía.In: Reflexión Académica En Diseño y Comunicación, Buenos Aires, v. 25, p.146-148, ago. 2015.2 Disponível em: <http://fido.palermo.edu/servicios_dyc/publicacionesdc/archivos/536_libro.pdf>. Acesso em: 24 set. 2017.

MORGADO-AGUIRRE, B.; LOPEZ-MARTIN, E.; CONESA-TEJADA, S. El uso de las TIC en la enseñanza universitaria de la fotografía. Primeros resultados del proyecto de innovación docente de la Universidad de Murcia. Arte, Individuo y Sociedad, v. 27, n. 2, p. 327-351, 2015

Newbury, Darren. Talking about practice: Photography students, photographic culture and professional identities. British Journal of Sociology and Education, v. 18, n. 3, p. 421-34, 1997.

PHUNGSUK, R.; VIRIYAVEJAKUL, C.; RATANAOLARN, T. Development of a problem-based learning model via a virtual learning environment. Kasetsart Journal of Social Sciences, v. 38, n. 3, p. 297- 
306, 2017.

PROQUEST. Disponível em: < http://www.proquest.com/LATAM-PT/> Acesso em: 18 dez. 2017.

RIVERA, C. D. Enseñanza de la fotografía en ambientes virtuales de aprendizaje. In: Undécima Conferencia Iberoamericana en Sistemas, Cibernética e Informática, Anais... 2012.

ROBBIE, D.; ZEENG, L. IT's evolving, they're changing, we're listening: everybody's learning. In: ASCILITE 2008 - The Australasian Society for Computers in Learning in Tertiary Education, p. 809818, Anais... 2008.

ROBBINS, J. Photographic education in the digital age. RPS Journal, v5, p. 239-41, 2000.

RUBINSTEIN, Daniel. Towards Photographic Education. Photographies, [s.I.], v. 2, n. 2, p.135-142, 8 set. 2009.

Disponível em:

https://www.academia.edu/528410/Towards_Photographic_Education>. Acesso em: 10 nov. 2017.

SAMPAIO, R. F.; MANCINI, M. C. Estudos de revisão sistemática: um guia para síntese criteriosa da evidência científica. Revista Brasileira de Fisioterapia, São Carlos, v. 11, n. 1, p.83-89, fev. 2007. Disponível em: < http://www.scielo.br/pdf/rbfis/v11n1/12.pdf>. Acesso em: 02 mar. 2018.

SANTAELLA, Lúcia. Linguagens líquidas na era da mobilidade. São Paulo: Paulus, 2014.

SCOPUS. Disponível em: <http://www.scopus.com> Acesso em: 18 dez. 2017.

SEMESP, Sindicato das Mantenedoras de Ensino Superior (Ed.). Diretrizes de Política Pública para - Ensino Superior Brasileiro 2017. São Paulo, 2017. Disponível em: <http://www.semesp.org.br/wp-content/uploads/2017/09/Diretrizes-de-Política-08-08-.pdf>. Acesso em: 3 fev. 2018.

SOBRAL, João Eduardo Chagas. A linguagem fotográfica na formação do designer em um ambiente de convergência tecnológica. 2011. 145 f. Tese (Doutorado) - Curso de Pós-graduação em Design, Departamento de Artes e Design do Centro de Teologia e Ciências Humanas, Pontifícia Universidade Católica do Rio de Janeiro - Puc-rio, Rio de Janeiro, 2011. Disponível em: <https://www.maxwell.vrac.puc-rio.br/Busca_etds.php?strSecao=resultado\&nrSeq=21687@1>. Acesso em: 24 set. 2017.

SUKSAI, P. The Effectiveness and the Comparison of e-Learning Lessons Efficiency on the Principles of Digital Photography in Technology of Photography Course. International Journal of Information and Education Technology, v. 6, n. 8, p. 598-602, 2016.

TORI, Romero. Educação sem distância: as tecnologias interativas na redução de distâncias em ensino e aprendizagem. São Paulo: Senac São Paulo, 2010. 254 p.

TRANFIELD, D.; DENYER, D.; SMART, P. Towards a Methodology for Developing Evidence-Informed Management Knowledge by Means of Systematic Review. British Journal of Management, v. 14. p. 207-222, 2003. Disponível em: <http://onlinelibrary.wiley.com/doi/10.1111/14678551.00375/epdf>. Acesso em: 24 jan 2018.

VOISARD, R.; CHAMPOD, C.; FURRER, J.; CURCHOD, J.; VAUTIER, A.; MASSONNET, G.; BUZZINI, P. Nicephor[e]: A web-based solution for teaching forensic and scientific photography. Forensic Science International, v. 167, n. 3, p. 196-200, 2007. 
WEB OF SCIENCE. Disponível em: <http://www.webofknowledge.com>. Acesso em: 18 dez. 2017. WEBSTER, Jane; WATSON, Richard T. Analyzing the past to prepare for the future: Writing a literature review. MIS quarterly, v.26, n.2, pp.13-23, 2002. Disponível em: < https://edisciplinas.usp.br/pluginfile.php/4031558/mod_resource/content/1/ANALYZING\%20THE \%20PAST\%20TO\%20PREPARE\%2OFOR\%20THE\%20FUTURE-

\%20WRITING\%20A\%20LITERATUR.pdf>. Acesso em: 22 jan. 2018.

ZHANG, Y.; HAO, X.; WANG, J.; JIAO, W.; DAI, W. The Thinking of Integrating between Informational Technology and Photography Course. International Journal of Digital Content Technology and its Applications, v. 7, n. 2, p. 487-494, 2013. 